\title{
THE DESIGN OF TERNARY PERTURBATION SIGNALS FOR LINEAR SYSTEM IDENTIFICATION
}

\author{
H. A. Barker', A. H. Tan ${ }^{2}$ and K. R. Godfrey ${ }^{3}$ \\ 1: School of Engineering, University of Wales Swansea, Swansea, SA2 8PP, U.K. \\ 2: Faculty of Engineering, Multimedia University, 63100 Cyberjaya, Malaysia. \\ 3: School of Engineering, University of Warwick, Coventry, CV4 7AL, U.K.
}

\begin{abstract}
A method is described for designing ternary perturbation signals to reduce the effects of both nonlinear distortion and noise in the identification of linear systems. This is achieved by suppressing harmonic multiples of 2 and 3 in the signals and optimizing three performance indices. Methods are given for designing primitive ternary signals to meet these criteria, and results are tabulated for signals with periods up to 78. It is then shown that these signals can be used to design pseudorandom ternary signals with longer periods that also meet the criteria. Results are tabulated for these signals and illustrated by an example. Copyright (C) 2005 IFAC
\end{abstract}

Keywords: Linear systems; Maximum-length sequences; Multilevel signals; Perturbation signals; Pseudorandom signals; System identification.

\section{INTRODUCTION}

In many identification experiments, it is required to identify the linear behaviour of a system in the presence of both nonlinear distortion and noise. For such experiments, it is therefore desirable that the perturbation signals used are designed to minimize these effects. In this paper, the design of ternary signals for this purpose is considered. Although these are the simplest kind of multilevel signal, they have unique advantages that make them useful for linear system identification. One advantage is that they are easily transduced to form a system input, making them particularly suitable for the identification of prototype systems. Important measurements often need to be made on industrial plant at an early stage of development, when input actuation is primitive, and multilevel signals with a small number of levels are ideal for this. Barker and Godfrey (1999) described an industrial application in which a prototype system for the stabilization of steel strip during galvanizing was identified by using electromagnets on each side of the strip. By applying a voltage to either electromagnet, or to neither, a ternary perturbation signal was generated.
Design criteria for ternary perturbation signals are developed in Section 2 of this paper, first for the reduction of nonlinear distortion, and then for the reduction of noise. Methods for obtaining primitive ternary signals to satisfy these criteria are then developed in Section 3. It is shown in Section 4 that most of these primitive signals may be used to generate pseudorandom ternary signals, thus creating a considerably larger collection of perturbation signals for the user. An example of a typical application of these ternary perturbation signals is given in Section 5. Data for immediate application of the results are provided in the form of design tables.

\section{NONLINEAR DISTORTION AND NOISE}

Reduction of the effects of nonlinear distortion on the accuracy of identification of the linear component of a system is achieved by choosing a perturbation signal with appropriate characteristics. The effects of distortion due to even-order nonlinearities may be completely eliminated by choosing an inverse-repeat perturbation signal, for which the period is even and 
even harmonics are zero (Godfrey, 1993). For a signal $u(i)$ with period $N$, the condition for this is

$$
u(i)+u(i+N / 2)=0 \quad \text { for all } i
$$

The effects of distortion due to odd-order nonlinearities cannot be eliminated, but they can be reduced by choosing an inverse-repeat perturbation signal for which the period is a multiple of 6 and harmonic multiples of 2 and 3 are zero, and the condition for this is

$$
u(i)+u(i+N / 3)+u(i+2 N / 3)=0 \quad \text { for all } i
$$

Only multilevel signals for which the nonzero levels are symmetrical about the zero level satisfy these conditions, and an example given by Barker, et al. (2004) shows how effective they are for reducing the effects of nonlinear distortion.

Reduction of the effects of noise on the accuracy of identification of the linear component of a system is achieved by choosing a perturbation signal with high performance indices in both the time domain and the frequency domain. The time domain index used here is PIPS, the Performance Index for Perturbation Signals, described by Godfrey, et al. (1999). PIPS is independent of the signal mean and amplitude. It is $100 \%$ for signals with the best possible performance, which are binary signals with equal occurrences of the two levels, and less for other signals. For signals that satisfy Equations 1 and 2, PIPS is given by

$$
\text { PIPS }=\frac{100}{\max u(i)}\left(\frac{2}{N} \sum_{i=1}^{N / 2} u^{2}(i)\right)^{1 / 2} \%
$$

The frequency domain index used here is EMIN, derived from the measure used by Kollár (1994) in the MATLAB Frequency Domain System Identification Toolbox. EMIN is also independent of the signal mean and amplitude. It is $100 \%$ for signals with the best possible performance, which in this case are signals with a uniform spectrum, and less for other signals. For signals that satisfy Equations 1 and 2, EMIN is given by

$$
\mathrm{EMIN}=\frac{100 \min |U(k)|_{k=1,5,7,11,13, \ldots} \%}{\left(\frac{6}{N} \sum_{k=1,5,7,11,13, \ldots}^{N / 2}|U(k)|^{2}\right)^{1 / 2} \%}
$$

where $U(k)$ is the Discrete Fourier Transform (DFT) of $u(i)$, defined as

$$
\begin{aligned}
U(k)=\frac{1}{N} \sum_{i=1}^{N} u(i) \exp \left(-\frac{2 \pi j i k}{N}\right) \\
\quad k=0,1, \ldots, N-1
\end{aligned}
$$

For a multilevel signal, PIPS and EMIN cannot both be $100 \%$, and the best compromise of performance is achieved when their product is maximised. This minimises a further performance index $\mathrm{TF}$, derived from the Time Factor used by Pintelon and Schoukens (2001). Using a development similar to that in Godfrey, et al. (2003), TF is given by

$$
\mathrm{TF}=0.5\left(\frac{10^{4}}{\mathrm{PIPS} \times \mathrm{EMIN}}\right)^{2}
$$

For signals with the best possible performance, when both PIPS and EMIN are $100 \%$, TF has its least possible value of 0.5 , as compared with 1 for a single sine wave. Ternary perturbation signals that satisfy Equations 1 and 2, and for which the value of TF is least for a given period $N$, are described as optimal ternary signals in this paper.

\section{PRIMITIVE TERNARY SIGNALS}

The simplest multilevel signals that satisfy the conditions in Equations 1 and 2 are ternary signals, which normally have a higher PIPS than signals with more than three levels and are much easier to design. Equations 1 and 2 show that all the members in a period of the signal are defined by the members in any third of a period. For signals that satisfy Equation 1, PIPS, EMIN and TF may be obtained from the members in any half of a period. A direct method for signal design is therefore:

1. Compute all permutations of the member values 1,0 and -1 for a third of a period.

2. For each permutation compute the member values 1,0 and -1 for a half of a period.

3. Compute PIPS, EMIN and TF for the signal.

4. Tabulate signals for which PIPS and EMIN are highest and TF is lowest.

Signals obtained by this method are the simplest examples of ternary signals that best satisfy all the criteria for reducing the effects of nonlinear distortion and noise. They are therefore described as primitive ternary signals. Primitive ternary signals with periods from 6 to 60 that have been obtained by this method are shown in the upper part of Table 1.

An alternative method is to compute the primitive ternary signals as computer-optimized signals. A development of the program multilev, described by McCormack, et al. (1995), is most suitable for this. The program multilev_new, described by Tan and Godfrey (2004), can be used to obtain signals for which TF is lowest and either PIPS or EMIN is highest. With this method, the primitive ternary signals with periods 6 and 12 are the same as those in Table 1, and those with periods from 18 to 60 are the same as one of the pair of signals for each of these periods in Table 1. An advantage of the method is that it can give optimal signals with periods greater than 60 , which with the direct method requires long computational times. It will always give a signal that is close to the optimum, but not necessarily the optimal signal. Primitive ternary signals with periods from 66 to 78 that have been obtained by this method are shown in the lower part of Table 1. Repeated computations indicate that these signals may be considered optimal. For signals with periods greater than 78 , a large number of repeated computations 
would be required to obtain signals that might be considered optimal.

\section{PSEUDORANDOM TERNARY SIGNALS}

The results in Table 1 are useful for applications where ternary signals with relatively short periods can be used. The number of effective harmonics is a sixth of the signal period, so even with a period of 78 the frequency response of a system can be obtained at only 13 frequencies. Although adequate for adaptive control schemes, this is unlikely to be sufficient for detailed characterization of the system dynamics. In the industrial application described by Barker and Godfrey (1999), for example, a signal with a period of 6858 was needed to provide sufficient data to identify a system with twenty significant mechanical resonances.

Fortunately there is a method for using nearly all of the primitive ternary signals in Table 1 to obtain ternary signals with the same properties but much longer periods. The method can be used when the primitive ternary signal period is one less than a prime, or a power of a prime, and for this reason, from among the signals with periods from 6 to 78 in Table 1, only those with period 54 cannot be used. The remaining signals are all primitive pseudorandom ternary signals, as described by Barker (2004), for each of which the period $N$ is related to a Galois field $\mathrm{GF}(q)$ by $N=q-1$.

A Galois field $\mathrm{GF}(q)$ has $q$ field elements that may be represented either by the integers $0123 \ldots q-1$, or through the powers of a primitive field element $g$ as $01 g g^{2} \ldots g^{q-2}$. A linear recurrence relationship

$$
s_{q, n}(i)+c_{1} s_{q, n}(i-1)+\ldots+c_{n} s_{q, n}(i-n)=0
$$

defines a sequence $s_{q, n}(i)$ of elements of $\operatorname{GF}(q)$, for which the characteristic polynomial $f_{n}(\mathrm{D})$ is

$$
f_{n}(\mathrm{D})=1+c_{1} \mathrm{D}+\ldots+c_{n} \mathrm{D}^{n}
$$

As shown by Zierler (1959), if $f_{n}(\mathrm{D})$ is a primitive polynomial, for which $(-1)^{n} c_{n}$ is a primitive field element, then $s_{q, n}(i)$ is a maximum-length sequence in $\mathrm{GF}(q)$ with the greatest possible period

$$
N=q^{n}-1
$$

In a period of a maximum-length sequence $s_{q, n}(i)$, each field element occurs $q^{n-1}$ times, except for the element 0 , which occurs $q^{n-1}-1$ times. When $n=1$, a primitive maximum-length sequence $s_{q, 1}(i)$ is generated, with period $N=q-1$ and characteristic polynomial $f_{1}(\mathrm{D})=1+c_{1} \mathrm{D}$. A period of $s_{q, 1}(i)$ is therefore $1 g g^{2} \ldots g^{q-2}$, where $g=-c_{1}$ is a primitive field element, so each field element occurs once, except for the element 0 , which does not occur.

A pseudorandom signal $u_{q, n}(i)$ is generated from the maximum-length sequence $s_{q, n}(i)$ by converting the field elements $01 g^{2} \ldots g^{q-2}$ into the signal levels $\mathrm{u}(0) \mathrm{u}(1) \mathrm{u}(g) \mathrm{u}\left(g^{2}\right) \ldots \mathrm{u}\left(g^{q-2}\right)$. Although the field elements are by definition distinct, it is not necessary that the signal levels are also distinct, and if they are restricted to 1,0 and -1 then a ternary pseudorandom signal is generated. The field element conversions define properties that are the same for all pseudorandom signals $u_{q, n}(i)$ generated from $s_{q, n}(i)$, including the primitive pseudorandom signals $u_{q, 1}(i)$ generated from $s_{q, 1}(i)$. Therefore

$$
u_{q, 1}(i)=\mathrm{u}\left(g^{i-1}\right) \quad \text { for } i=1,2, \ldots, q-1
$$

The conversions $u(1) u(g) u\left(g^{2}\right) \ldots u\left(g^{q-2}\right)$ of the nonzero field elements are therefore obtained from a period of the primitive pseudorandom signal $u_{q, 1}(i)$. The conversion $u(0)$ of the zero field element must be zero if the pseudorandom signal $u_{q, n}(i)$ is to satisfy Equations 1 and 2 when $n>1$, so $\mathrm{u}(0)=0$. For example, from Table 1 a primitive pseudorandom signal from $\mathrm{GF}(7)$ is $110-1-10$, and a primitive element of $\operatorname{GF}(7)$ is 3 , so from Equation $10 u(1)=1$, $\mathrm{u}(3)=1, \mathrm{u}(2)=0, \mathrm{u}(6)=-1, \mathrm{u}(4)=-1, \mathrm{u}(5)=0$. Together with $u(0)=0$, these define the conversions of all the field elements for which all pseudorandom signals $u_{q, n}(i)$ from $\mathrm{GF}(7)$ have the same properties as the primitive pseudorandom signal $u_{q, 1}(i)$.

Table 2 shows the field element conversions, obtained from the primitive pseudorandom signals $u_{q, 1}(i)$ in Table 1, for all pseudorandom ternary signals $u_{q, n}(i)$ from GF(7) to GF(79). The EMIN values for $u_{q, n}(i)$ are invariant as $n$ increases, but the PIPS values decrease as $n$ increases, because the proportion of $\mathrm{u}(0)$ values in a period of $u_{q, n}(i)$ increases. As $n$ becomes large, PIPS approaches its minimum value of $[(q-1) / q]^{1 / 2}$ times its value for $u_{q, 1}(i)$, and this is the value shown in Table 2. As $n$ becomes large, TF approaches its maximum value of $q /(q-1)$ times its value for $u_{q, 1}(i)$, and this is the value shown in Table 2. By comparing the PIPS and $\mathrm{TF}$ values in Tables 1 and 2, it can be seen that the decrease in PIPS and the increase in TF as $n$ increases are only small. The software GALOIS, described by Barker (2001), has facilities for obtaining all maximum-length sequences in $\mathrm{GF}(q)$ for $q \leq 128$, and for entering the field element 
conversions that generate pseudorandom signals from them. Although there is no theoretical limit to the periods of $u_{q, n}(i)$ that can be obtained by increasing $n$, periods of up to 7000 , with from 1 to over 1000 effective harmonics, are sufficient for most practical purposes. The periods of all signals obtainable from Table 1 that are less than 7000 are:

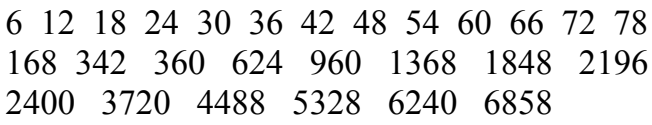

\section{EXAMPLE}

The example given here is a typical application of the ternary perturbation signals designed using the data in Tables 1 and 2, which is the identification of the linear component of a system that contains nonlinearities. The nature of the nonlinearities does not affect either the signal design or the identification method used, and in this case the nonlinearities are in the Hammerstein form. With the system input signal $u(t)$ applied to the part of the system that contains the nonlinearities, an intermediate inaccessible signal $v(t)$ is generated that is given by

$$
v(t)=u(t)+0.6 u^{2}(t)+0.2 u^{3}(t)+0.1 u^{4}(t)
$$

This signal is then the input to the linear part of the system with transfer function $\frac{1}{5 s+1}$, generating the system output signal $y(t)$. The $5 \mathrm{~s}$ time constant is to be identified from the digital signals $u(i)$ and $y(i)$, sampled from $u(t)$ and $y(t)$ at a rate of $1 \mathrm{~Hz}$.

Several pseudorandom ternary signals from Table 2 are suitable choices for the perturbation signal $u(t)$ in this case. The chosen signal is generated from a maximum-length sequence $s_{31,2}(i)$ in $\operatorname{GF}(31)$, with characteristic polynomial $f_{2}(\mathrm{D})=1+2 \mathrm{D}+3 \mathrm{D}^{2}$ and period $N=31^{2}-1=960$. Table 2 gives the field element conversions for which the resulting pseudorandom ternary signal $u_{31,2}(i)$ is optimal, and these conversions are used in this case to generate a signal for which PIPS is $80.4 \%$, EMIN is $89.4 \%$ and $\mathrm{TF}$ is 0.97 .

The time constant of the system is identified from single steady-state periods of $u(i)$ and $y(i)$, and their DFTs $U(k)$ and $Y(k)$. These are shown in Fig. 1. Estimates of the time constant are obtained from the ratios $Y(k) / U(k)$ at the nonzero harmonics of the input signal, using the routine elis from the MATLAB Frequency Domain System Identification Toolbox (Kollár, 1994). The results obtained for three cases with differing signal to noise ratios (SNR) are:
1. No Noise
Time Constant $=5.000 \mathrm{~s}$
2. $\mathrm{SNR}=30 \mathrm{~dB}$
Time Constant $=5.007 \mathrm{~s}$
3. $\mathrm{SNR}=15 \mathrm{~dB}$
Time Constant $=5.042 \mathrm{~s}$

The results obtained are excellent due to the welldesigned spectrum of the perturbation signal, which has harmonic multiples of 2 and 3 suppressed.

\section{CONCLUSIONS}

Ternary perturbation signals are particularly suitable for the identification of linear systems in the presence of both nonlinear distortion and noise. They are the simplest form of signal for which harmonic multiples of 2 and 3 can be suppressed, thus allowing the effects of even-order nonlinearities to be completely eliminated, and the effects of odd-order nonlinearities to be reduced. For signals designed to have these characteristics, the effects of noise may be reduced by maximizing the time domain performance index PIPS and the frequency domain performance index EMIN. As PIPS and EMIN cannot both be $100 \%$ for a ternary signal, the best performance is obtained when their product is maximized, as this minimizes a third performance index TF.

Two methods for designing primitive ternary signals to meet these criteria have been described. The first is a direct method that gives definitive results for signals with periods up to 60 . The second is a computer-optimized method that gives additional results for signals with periods up to 78 . These results have been tabulated for design purposes. It has then been shown that these primitive ternary signals can be used to design pseudorandom ternary signals with much longer periods, thus providing a much larger set of perturbation signals. These results have also been tabulated for pseudorandom ternary signals from Galois fields up to GF(79).

An example has been given that shows how effective these signals are for reducing the effects of nonlinear distortion and noise in the identification of linear systems.

\section{REFERENCES}

Barker, H. A. and K. R. Godfrey (1999). System identification with multi-level periodic perturbation signals, Control Engineering Practice, 7, 717-726.

Godfrey, K. R. (1993). Perturbation Signals for System Identification, Prentice-Hall, Hemel Hempstead.

Barker, H. A., A. H. Tan and K. R. Godfrey (2004). Design of multilevel perturbation signals with harmonic properties suitable for nonlinear system identification, IEE Proc.-Control Theory Appl., 151, 145-151.

Godfrey, K. R., H. A. Barker and A. J. Tucker (1999). Comparison of perturbation signals for linear identification in the frequency domain, IEE Proc.-Control Theory Appl., 146, 535-548. 
Kollár, I. (1994). Frequency Domain System Identification Toolbox for use with MATLAB, The MathWorks, Natick, MA.

Pintelon, R. and J. Schoukens (2001). System Identification: A Frequency Domain Approach, IEEE Press, New York.

Godfrey, K. R., A. H. Tan and H. A. Barker (2003). A survey of readily accessible perturbation signals, Proc. $13^{\text {th }}$ IFAC Symposium on System Identification (SYSID 2003), Rotterdam, Netherlands, 653-658.

McCormack, A. S., K. R. Godfrey and J. O. Flower (1995). Design of multilevel multiharmonic signals for system identification, IEE Proc.Control Theory Appl., 143, 247-252.
Tan, A. H. and K. R. Godfrey (2004). An improved routine for designing multi-level multi-harmonic signals, Proc. UKACC Conference Control 2004, (M. N. Sahinkaya and K. A. Edge, Eds.) ISBN 0 86197130 2, Bath, UK, Paper ID-027.

Barker, H. A. (2004). Primitive maximum-length sequences and pseudo-random signals, Trans. InstMC, 26, 339-348.

Zierler, N. (1959). Linear recurring sequences, $J$. Soc. Indust. Appl. Math., 7, 31-48.

Barker, H. A. (2001). GALOIS - a program for generating pseudo-random perturbation signals, Proc. $12^{\text {th }}$ IFAC Symposium on System Identification (SYSID 2000), (R. Smith, Ed.), Elsevier Science, Oxford, 505-508.
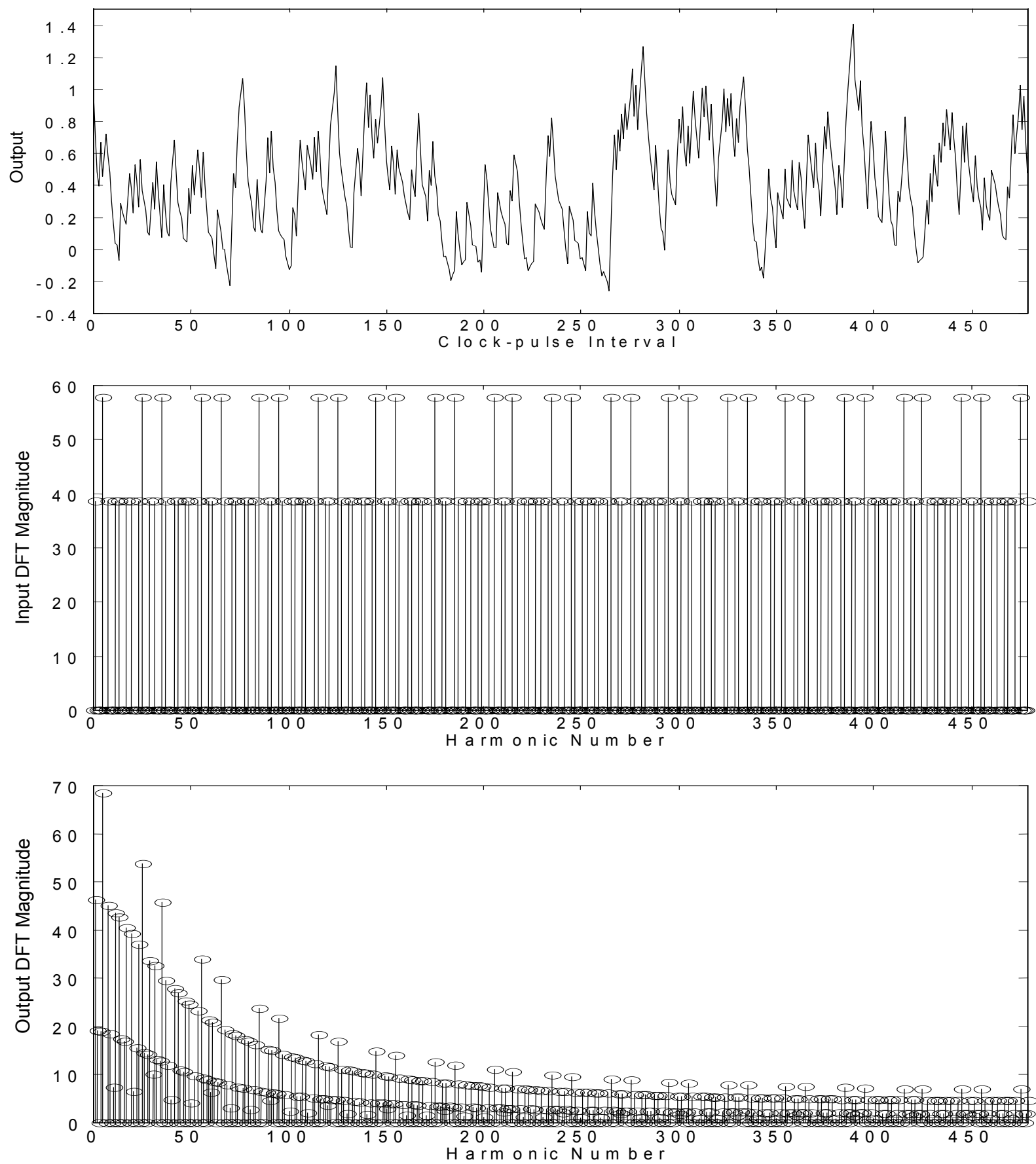

Fig. 1 Half periods of signals and their discrete Fourier transforms used in the Example 
Table 1 Primitive ternary signals with harmonic multiples of 2 and 3 suppressed

\begin{tabular}{ccccl}
$\begin{array}{c}\text { Period } \\
N\end{array}$ & $\begin{array}{c}\text { PIPS } \\
\%\end{array}$ & $\begin{array}{c}\text { EMIN } \\
\%\end{array}$ & TF & First half of primitive ternary signal. Second half is inverse of first half \\
\hline 6 & 81.6 & 100 & 0.75 & ++0 \\
12 & 81.6 & 100 & 0.75 & $++0+-0$ \\
18 & 81.6 & 70.0 & 1.53 & $++000+--+$ \\
18 & 47.1 & 100 & 2.25 & $+00+00000$ \\
24 & 70.7 & 100 & 1.00 & $++00+0+00-+0$ \\
24 & 81.6 & 80.4 & 1.16 & $+++0+00+0--+$ \\
30 & 73.0 & 100 & 0.94 & $+++000+00+-0-0+$ \\
30 & 81.6 & 89.4 & 0.94 & $+++000+0++-0-++$ \\
36 & 81.6 & 78.3 & 1.22 & $++++0-+0+0+00-0-++$ \\
36 & 47.1 & 100 & 2.25 & $+00+00000+00-00000$ \\
42 & 81.6 & 92.6 & 0.88 & $+++00+00+0-+++-0--+0+$ \\
42 & 61.7 & 100 & 1.31 & $++00000+0+00+00-+00+0$ \\
48 & 76.4 & 100 & 0.86 & $+++0+00-++0+00+000-+-0++$ \\
48 & 81.6 & 84.4 & 1.05 & $+++00000+0++-+++0-0+-+++$ \\
54 & 81.6 & 86.6 & 1.00 & $+++++0-+-+00++--+00--00-00+$ \\
54 & 27.2 & 100 & 6.75 & $+00000000+00000000000000000$ \\
60 & 81.6 & 94.9 & 0.83 & $+++0-+0-0-+00-00+0--0---+-++-0$ \\
60 & 51.6 & 100 & 1.88 & $+0000000+0+0+0-000+000+0-00000$ \\
66 & 81.6 & 90.9 & 0.91 & $++++000+---+0+0-+-000-0-0--+--++0$ \\
72 & 81.6 & 88.0 & 0.97 & $++++0-++-0+00000+0++-+0+----++000+-+$ \\
78 & 81.6 & 96.1 & 0.81 & $++0+-0++0+-0-+0-+0++0-+0--0--0++0--0+-0$
\end{tabular}

Table 2 Field element conversions for pseudorandom ternary signals

Field Period PIPS EMIN TF

\begin{tabular}{|c|c|c|c|c|c|}
\hline $\mathrm{GF}(q)$ & $N$ & Min \% & $\%$ & Max & Conversions $\mathrm{u}(0), \mathrm{u}(1), \ldots, \mathrm{u}(q-1)$ of field elements $0,1, \ldots, q-1$ \\
\hline 7 & $7^{n}-1$ & 75.6 & 100 & 0.87 & $0+0+-0-$ \\
\hline 13 & $13^{n}-1$ & 78.4 & 100 & 0.81 & $0++-0-00+0+--$ \\
\hline 19 & $19^{n}-1$ & 79.5 & 70.0 & 1.62 & $0++00+--0+-0++-00--$ \\
\hline 19 & $19^{n}-1$ & 45.9 & 100 & 2.37 & $0+000000+00-000000-$ \\
\hline 25 & $25^{n}-1$ & 69.3 & 100 & 1.04 & $0+-+-+0+00000+-0+-00-00-0$ \\
\hline 25 & $25^{n}-1$ & 80.0 & 80.4 & 1.21 & $0+00-+0+00-+++++-----00-0$ \\
\hline 31 & $31^{n}-1$ & 71.8 & 100 & 0.97 & $0+-+00+0-++00000-+00000--+0-00-+-$ \\
\hline 31 & $31^{n}-1$ & 80.3 & 89.4 & 0.97 & $0+-+00+--++-000-+000+--++-00-+-$ \\
\hline 37 & $37^{n}-1$ & 80.5 & 78.3 & 1.26 & $0++-++00++-0-0+-00+-00+-0+0+--00--+--$ \\
\hline 37 & $37^{n}-1$ & 46.5 & 100 & 2.31 & $0+0000-0+00+00000000000000-00-0+0000-$ \\
\hline 43 & $43^{n}-1$ & 80.7 & 92.6 & 0.90 & $\begin{array}{l}0+0++00+-+-0+-+-0+-0+0 \\
\rightarrow 0-0+-0+-+-0+-+-00--0-\end{array}$ \\
\hline 43 & $43^{n}-1$ & 61.0 & 100 & 1.34 & $\begin{array}{l}0+0++0-0000-0000000+-+ \\
\rightarrow-+-0000000+0000+0--0-\end{array}$ \\
\hline 49 & $49^{n}-1$ & 75.6 & 100 & 0.88 & $\begin{array}{l}0+-00+-+-000+---+00++0++0 \\
\rightarrow 0-000+00--+--00-+-+-000+\end{array}$ \\
\hline 49 & $49^{n}-1$ & 80.8 & 84.4 & 1.07 & $\begin{array}{l}0+-00+-+0+000-0-++00+++++ \\
\rightarrow--+--++---0-00--+-+000-0\end{array}$ \\
\hline 61 & $61^{n}-1$ & 81.0 & 94.9 & 0.85 & $\begin{array}{l}0++0+--+00-00-0--0-++++--00-+-0 \\
\rightarrow 0+-+00++----+0++0+00+00-++-0--\end{array}$ \\
\hline 61 & $61^{n}-1$ & 51.2 & 100 & 1.91 & $\begin{array}{l}0+000+000+00+-000000-0000+0+000 \\
\rightarrow 000-0-0000+000000+-00-000-000-\end{array}$ \\
\hline 67 & $67^{n}-1$ & 81.0 & 90.9 & 0.92 & $\begin{array}{l}0++0+---+0+++00+0-+--+--++00--0000 \\
\rightarrow 0000++00--++-++-+0-00---0-+++-0--\end{array}$ \\
\hline 73 & $73^{n}-1$ & 81.1 & 88.0 & 0.98 & $\begin{array}{l}0+-+++0+-00-0-++0+-+0-+-0+0+0+000++-+ \\
\rightarrow-+--000-0-0-0+-+0-+-0--+0+00+-0---+-\end{array}$ \\
\hline 79 & $79^{n}-1$ & 81.1 & 96.1 & 0.82 & $\begin{array}{l}0+-+0000-0+0+--++-+0-++0+-0+-0+00----++0 \\
\rightarrow 0--++++00-0+-0+-0--+0-+--++-0-0+0000-+-\end{array}$ \\
\hline
\end{tabular}

